\section{MECHANISM OF TETRACYCLINE \\ RESISTANCE IN PSEUDOMONAS AERUGINOSA CARRYING AN R FACTOR}

\author{
Koji O'hara and Megumi Kono \\ Department of Microbiology, \\ Tokyo College of Pharmacy, \\ 1-10-19, Ueno-sakuragi, Taito-ku, \\ Tokyo 110, Japan
}

(Received for publication February 10, 1975)

Resistance to tetracycline (TC) is common in enteric bacteria and the biochemical mechanism of resistance to $\mathrm{TC}$ has been investigated by many workers. ${ }^{1 \sim 7)}$ A report by TSENG and BRYAN is the only description of the mechanism of TC-resistance in Pseudomonas aeruginosa carrying an $\mathrm{R}$ factor, R931. ${ }^{8}$ ) During a survey of drug resistance in $P$. aeruginosa, we found several $\mathrm{R}$ factors from such clinical isolates. One of the $\mathrm{R}$ factors, kR102, was different from R931 by the following genetic traits: firstly, kR102 has five resistance determinants, TC-chloramphenicol-streptomycinsulfonamide-kanamycin, and R931 has two, TC-streptomycin. Secondly, a streptomycininactivating enzyme in the former has not been demonstrated, even though the MIC of the bacterial strain carrying kR102 is very high, over $25,000 \mathrm{mcg} / \mathrm{ml}$ (unpublished data), while a streptomycin-phosphorylating enzyme has been detected in the bacteria carrying R931 (MIC 1,600 mcg/ml). Neither $\mathrm{R}$ factor has been successfully transferred to enteric bacteria. This paper deals with the mechanism

Fig. 1.

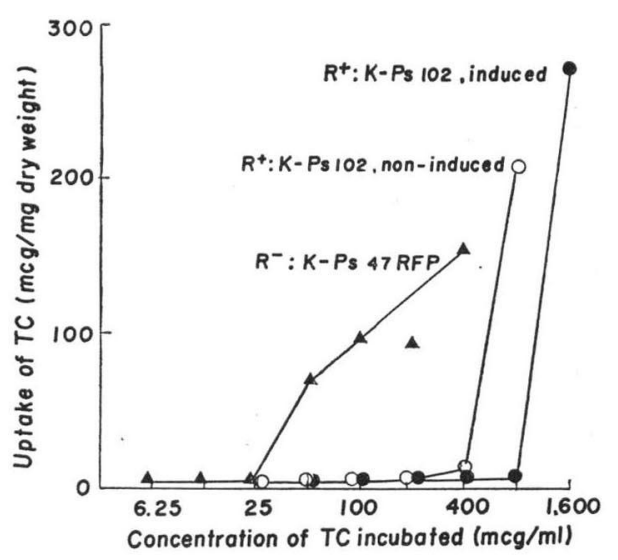

of kR102 mediated resistance to TC.

$P$. aeruginosa, K-Ps 47 RFP, was used as $\mathrm{R}^{-}$strain, a clinically-isolated rifampicin resistant mutant of K-Ps 47 sensitive to the drugs mentioned above. The minimum inhibitory concentration (MIC) of each strain to $\mathrm{TC}$ was determined by the agar dilution method and estimated as $800 \mathrm{mcg} / \mathrm{ml}$ to K-Ps 102 and $50 \mathrm{mcg} / \mathrm{ml}$ to $\mathrm{K}-\mathrm{Ps} 47 \mathrm{RFP}$, respectively.

Uptake of TC was determined according to the method of Sompolinsky et al. ${ }^{8)}$ When the concentration of TC used in uptake studies exceeded $25 \mathrm{mcg} / \mathrm{ml}$, the TC-uptake of K-Ps 47 RFP increased rapidly. By contrast, an increase of TC-uptake in the resistant strain K-Ps 102 was not observed even at concentrations up to $200 \mathrm{mcg} / \mathrm{ml}$. However, when the external concentration of TC exceeded 400 $\mathrm{mcg} / \mathrm{ml}$, the slope of the uptake curve sharply increased. The concentration of $\mathrm{TC}$, at which a rapid increase occurred, corresponded with the MIC for each strain. This suggests that at these concentrations, rapid entry of the drug into cells causes inhibition of cell growth.

The effect of pretreatment of cells with TC on drug resistance was examined by determining bacterial growth at various concentrations of TC by the method previously reported. ${ }^{9)}$ And it was found that the resistant strain K-Ps 102 could grow in broth containing 400 $\mathrm{mcg} / \mathrm{ml}$ of $\mathrm{TC}$ after treatment with 12.5 $\mathrm{mcg} / \mathrm{ml}$ of $\mathrm{TC}$ for 30 minutes; without the pretreatment complete inhibition of the strain was observed. The MIC in the induced population was $1,600 \mathrm{mcg} / \mathrm{ml}$ of TC and the TC-uptake of the induced population of K-Ps 102 was less than that of a noninduced population of the same strain, as Fig. 1 shows. The TC-resistance of TC-induced cells of K-Ps 102 decreased on growth in TC-free medium. From these results, it was concluded that the TC-resistance of $\mathrm{R}^{+}$strain K-Ps 102 is inducible and that $\mathrm{TC}$ is an active inducer; we presume that the induced resistance is due to decreased uptake of the drug.

TC was extracted from cells of culture of K-Ps 102 which had been exposed to the drug, and subjected to spectrophotometric assay and microbioassay according to the method of Sompolinsky et al. ${ }^{8)}$ The results indicated that the extracted TC showed the 
same absorption spectrum and antibacterial activity as those of authentic TC. In addition, possible inactivation of $\mathrm{TC}$ by cell-free extracts prepared from resistant bacteria was examined according to the method reported previously, ${ }^{10}$ no reduction in antibacterial activity of the drug occurred.

K-Ps 47 RFP (kR102) was obtained from K-Ps 47 RFP by mating with K-Ps 102. The conjugant had acquired the resistance markers of the donor. TC-uptake in this strain, K-Ps 47 RFP (kR102), with or without pretreatment with TC was examined and it was shown that the TC-uptake curves in both cases were nearly the same as that of the induced population of K-Ps 102. The differences between strains K-Ps 102 and K-Ps 47 RFP (kR102) may be due to the differences in expression of the $\mathrm{R}$ factor in the two different host strains.

Spheroplasts of strain K-Ps 102 became sligthtly sensitive to TC compared with that of intact cells of the same strain. However, the level of resistance in the spheroplasts was higher than that of the $\mathrm{R}^{-}$strain K-Ps 47 RFP (unpublished data). This implies that the mechanism of $\mathrm{TC}$-uptake in $\mathrm{R}^{+}$strains might be due to an enzyme, which is located at cell membrane and which is responsible for the active transport of TC into the cell. This discussion agrees with the report of LEVY et $a l .{ }^{7)}$ in which it has been shown that TC specific membrane bound protein was detected in $\mathrm{R}^{+}$cells, but not in $\mathrm{R}^{-}$cells.

In summary, the report indicates that there is a clear correlation between the MIC of a strain and its TC-uptake; this confirms the report by TSENG and BRYAN. ${ }^{6)}$ Studies on the mechanisms of resistance to other antibiotics determined by the R factor, kR102, are now under way.

\section{References}

1) IZAKi, K. \& K. ARIma: Disappearance of oxytetracycline accumulation in the cells of multiple drug-resistant Escherichia coli. Nature 200: 384 385, 1963

2) Izaki, K.; K. KiUchi \& K. Arima: Specificity and mechanism of tetracycline resistance in a multiple drug resistant strain of Escherichia coli. J. Bact. 91: 628 633, 1966

3) Franklin, T.J. \& A. Godfrey: Resistance of Escherichia coli to tetracyclines. Biochem. J. 94: $54 \sim 60,1965$

4) Unowsky, J. \& M. Rachmeler: Mechanisms of antibiotic resistance determined by resistance-transfer factors. J. Bact. 92: 358 365, 1966

5) Franklin, T. J.: Resistance of Escherichia coli to tetracyclines. Biochem. J. 105: 371 378, 1967

6) Tseng, J. T. \& L. E. Bryan: Mechanisms of $\mathrm{R}$ factor R931 and chromosomal tetracycline resistance in Pseudomonas aeruginosa. Antimicr. Agents \& Chemoth. 3: 638 641, 1973

7) Levy, S. B. \& L. McMurry: Detection of an inducible membrane protein associated with R-factor-mediated tetracycline resistance. Biochem. Biophys. Res. Commun. 56: 1060 1068, 1974

8) Sompolinsky, D; Y. Zaidenzaig, R. Zieglerschlomowitz \& N. Abramova: Mechanism of tetracycline resistance in Staphylococcus aureus. J. Gen. Microbiol. 62: 351 362, 1970

9) Kono, M.; K. O'hara, M. Honda \& S. MitsuHASHI: Drug resistance of Staphylococci. XI. Induction of chloramphenicol resistance by its derivatives and analogues. J. Antibiotics 22: 603 607, 1969

10) O'hara, K.; M. Kono \& S. Mitsuhashi: Enzymatic inactivation of a new aminoglycoside antibiotic, sisomicin, by resistant strains of Pseudomonas aeruginosa. Antimicr. Agents \& Chemoth. 5: 558 561, 1974 